\title{
In-hospital mobilization after cardiac surgery: investigation of a dose-effect of once- and twice-daily exercise on cardiac autonomic modulation
}

Renata Gonçalves Mendes* (1); Camila Bianca Falasco Pantoni1 ${ }^{1,2}$ (1); Rodrigo Polaquini Simões ${ }^{1}$ (D); Luciana Ditomaso-Luporini1,3 (1); Flávia Cristina Rossi Caruso Bonjorno ${ }^{1}$ (1);

Erika Zavaglia Kabbach¹ (D); Audrey Borghi-Silva ${ }^{1}$ (D)

\begin{abstract}
Background: Coronary artery bypass surgery (CABG) implies in impairment of cardiac autonomic modulation (CAM). In-hospital mobilization positively affects CAM; however, it is not known whether higher exercise dosage would provide the same benefits in CAM as lower dosage in patients post-CABG. Aims: To investigate the dose-effect of an exercise inpatient program performed once or twice-daily on cardiac autonomic modulation post-CABG. Design: Prospective trial with a quasiexperimental design. Methods: Thirty patients of both sexes with a diagnosis of coronary heart disease and first-time post-CABG were allocated in a non-random manner into GEX1 $(n=10)$ and GEX2 $(n=10)$, composed of patients engaged in one and two daily exercise sessions plus usual care (breathing exercises, education with no systematized mobilization exercise program), respectively; and control group (CG, $n=10)$, composed of those who only received usual care. Patients underwent a mobilization protocol until discharge, which included a progressive five steps of active-assistive exercises of lower/upper limbs. Heart rate (HR) and R-wave intervals (RRi) were recorded by heart rate monitor at basal condition and discharge time. CAM was assessed by heart rate variability (HRV). Results: At discharge, the higherdose group (GEX2) showed significant higher values of: parasympathetic (RMSSD: $7.9 \pm 1.3 \mathrm{~ms}$ vs $4.6 \pm 0.4 \mathrm{~ms}$ ) and overall CAM indexes (STD RR: $7.5 \pm 1.1 \mathrm{~ms}$ vs $4.8 \pm 0.5 \mathrm{~ms}$ and TINN ( $38 \pm 6.4 \mathrm{~ms}$ vs $23 \pm 2.8 \mathrm{~ms}$ ) compared with CG, respectively. In addition, mean HR (GEX2: $88.1 \pm 12.3$ bpm; GEX1: 79.4 \pm 5.7 bpm, CG: $100.4 \pm 10.1 \mathrm{bpm}$ ) and mean RR (GEX2: 694.0 \pm 101.8 bpm; GEX1: $759.1 \pm 57.0$ bpm, CG: 602.8 \pm 59.1 bpm) were different to exercise groups compared to controls regardless dosage. However, there was no significant difference between the exercise groups (GEX1 and GEX2). Conclusions: Regardless dosage, post-CABG patients who engaged in a mobilization-exercise program demonstrated better cardiac autonomic modulation at discharge compared with the control group. The results are encouraging further investigation in the field of in-hospital exercise rehabilitation dosage on cardiac autonomic modulation in a more comprehensive sample to strengthen the findings.
\end{abstract}

Keywords: Autonomic Nervous System; Coronary Artery Disease; Exercise; Rehabilitation; Cardiac Rehabilitation.

\section{How to cite}

Mendes RG, Pantoni CBF, Simões RP, Ditomaso-Luporini L, Bonjorno FCRC, Kabbach EZ, et al. In-hospital mobilization after cardiac surgery: investigation of a dose-effect of once- and twice-daily exercise on cardiac autonomic modulation. Cardiorespir Physiother Crit Care Rehabil. 2021;1:e42060. https://doi.org/10.4322/2675-9977.cpcr.42060

\section{How can the results of this study be used in clinical practice?}

- Inpatient exercise-based rehabilitation improves cardiac autonomic control in post-CABG.

- Once or twice daily exercise rehabilitation post-CABG results in heart rate variability benefits.

- The results strengthen the importance of inpatient exercise-based rehabilitation focusing on an important outcome that is the cardiac autonomic control.

\author{
CAAE 0191.0.135.000-09). \\ Clinical Trial Registration: The study was \\ registered in ensaiosclinicos.gov.br (REBEC)- \\ RBR-34khhv and in Trial RBR-5d877q \\ Accepted: February 21, 2021. \\ Study conducted at: Laboratório de \\ Fisioterapia Cardiopulmonar, Universidade \\ SP, Brasil and Irmandade da Santa Casa de \\ Misericórdia de Araraquara, Araraquara, SP, \\ Brasil. \\ Ethical approval: The protocol was approved \\ by the local Ethics Committee (numbers \\ Departamento de Fisioterapia, Laboratório \\ ( \\ Universidade Federal de São Carlos - UFSCar,

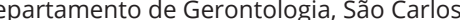 \\ SP, Brasi \\ Renata Gonçalves Mendes \\ CEP 13565-905, São Carlos (SP), Brasil \\ Tel.: +55 (16) 3306-6883
}

cc) (i) (2) (2)

Trial RBR-5d877q 


\section{Introduction}

Cardiac rehabilitation (CR) is strongly recommended for patients after coronary artery bypass grafting (CABG) surgery ${ }^{1}$. Early mobilization as part of an inpatient physical program is encouraged after cardiac surgery in order to counteract surgical and hospitalization negative effects ${ }^{2}$. Several benefits of exercise-based rehabilitation have been demonstrated, including improvement of functional capacity and reduction of postoperative complications, length of hospital stay ${ }^{3}$, and health care costs ${ }^{4}$.

Previously, our research group has already demonstrated beneficial effects of an early mobilization program, performed once-daily on cardiac autonomic modulation (CAM) in post $\mathrm{CABG}$ patients ${ }^{5,6}$. Interestingly, the dysfunction of the autonomic nervous system seems to be associated with increased risk of morbidity and mortality in these patients $\mathrm{s}^{7,8}$; therefore, CR represents a powerful tool to improve clinical care and patients' outcomes.

Although early mobilization has been recommended, studies on the optimal dose effect of exercise in a hospital setting on different outcomes has not yielded conclusive results. A Swedish survey indicates that physiotherapysupervised mobilization and exercise following cardiac surgery are prescribed ranging from one to three times a day during hospital stay ${ }^{9}$. However, there is no information on the benefits of different dosages of mobilization.

A cumulative effect of exercise or a dose-response relationship between physical exercises and health outcomes was previously reported and greater benefit has been linked with higher amounts of exercise ${ }^{10}$. Conversely, a low doseresponse relationship between dose-exercise therapy and improvements was also reported ${ }^{11}$. Nevertheless, the effect of different exercise dosage on CAM early after CABG has not been explored before. Therefore, to contribute to an adequate post-CABG rehabilitation management, the current study aimed to investigate the dose-effect of an exercise inpatient program performed once or twice-daily on cardiac autonomic modulation post-CABG. It was hypothesized a dose-dependent adaptation on CAM with greater benefit in the twice-daily exercise group.

\section{Methods}

\section{Population}

This study is a prospective trial with a blinded outcome assessment and a quasi-experimental design, conducted within the coronary unit and cardiovascular ward of a tertiary hospital in Araraquara- SP-Brazil. All methods were carried out in accordance with relevant guidelines and regulations (Declaration of Helsinki). Patients awaiting first-time $\mathrm{CABG}$ were invited to participate in the study, after giving written informed consent. The protocol was approved by the local Ethics Committee (numbers
197/2005 CAAE 0095.0.135.000-05; 109/2010 CAAE 0191.0.135.000-09).

Thirty patients undergoing elective CABG surgery were enrolled in three different groups: GEX1 $(n=10)$ and GEX2 $(n=10)$, were composed of patients who performed a physical exercise protocol (mobilization exercise program) once and twice-daily, respectively plus usual care (breathing exercises, education instruction and no systematized mobilization exercise program); and $\mathrm{CG}, \mathrm{n}=10$, performed only the usual care. This study was defined as a quasi-experimental design in which participants were assigned to either control group or experimental groups in a non-random manner. Eligibility criteria for participants included patients of both sexes who undergone an elective and first CABG carried out with cardiopulmonary bypass. Exclusion criteria comprised: emergent, concomitant surgery or other types of cardiac surgery, with recent myocardial infarction (less than six months), implanted pacemaker, unstable angina, chronic disturbances in heart rhythm, significant acute arrhythmias, valvular heart disease, chronic obstructive pulmonary disease, severe non-cardiac diseases, surgical complications, clinical instability, use of vasoactive drugs and inability to perform rehabilitation according to the proposed protocol.

Pre-operatively, patients' age, gender, weight, height, body mass index (BMI), cardiac risk factors and other relevant medical history were documented. Additionally, education about the effects of surgery on cardiorespiratory function, post-operative routines and the CR program were provided. Post-operatively, surgical and hospital data were recorded.

\section{Interventions}

During the inpatient phase, a daily-supervised usual care was applied to all patients. It consisted of respiratory exercises as: standard voluntary deep-breathing exercises from functional residual capacity to total lung capacity (40 deep breaths in four sets of 10, each breath included a $5 \mathrm{~s}$ hold at end of inspiration) followed by coughs or huffs (with wound support) for approximately $15 \mathrm{~min}$. Patients were also instructed to perform these breathing and coughing exercises independently every waking hour. Patients of control group received the usual care intervention.

Patients allocated to the exercise groups were engaged to one (GEX1) or two daily exercise sessions (GEX2) plus usual care. Exercise protocol was similar for both groups and initiated on the first post-operative day until discharge ${ }^{5}$. Heart rate (HR) was monitored and maintained around 20 beats per minute above resting value as described previously ${ }^{12}$, using a Polar S810i telemetry system (Polar Electro Oy, Kempele, Finland).

Mobilization exercise program: The protocol was organized in steps. Each step corresponded to one day of postoperative intervention. STEP 1: Active-assistive exercises of the lower/upper extremities - ankles and wrists, 5 sets of 10 repetitions; bed inclined at $45^{\circ}$; STEP 2: Active exercises of upper and lower limbs in sitting position $\left(90^{\circ}\right)$ - flexion- 
extension of the bilateral shoulder, elbow, wrist, knee and ankle; adduction-abduction of the hips ( 2 sets of 15 for each) and orthostatic position/walking on spot ( $5 \mathrm{~min}$ ) as tolerated; STEP 3: Active exercises, as in step 2, in 3 sets of 15 in the sitting position and ambulation within the inpatient ward (5 min) (approximate time spent $=50 \mathrm{~min}$ ); STEP 4: Exercises similar in step 3 in sitting position; ambulation within the inpatient ward (10 min); STEP 5: Exercises similar to step 3 in orthostatic position, ambulation $(10 \mathrm{~min})$ and flight of stairs (four steps). STEP 5 was remained to those hospitalized beyond five days.

Interventions were undertaken by physiotherapists, specifically trained in the methods used in the study; the hospital staff were asked to avoid discussing issues of physiotherapy with patients, and to refer any questions regarding the study or physiotherapy treatments to the research team.

\section{Outcomes}

The main outcome of this study was the CAM measured by heart rate variability (HRV), which is a considerable potential tool to assess the autonomic nervous system in patients with cardiovascular disorders ${ }^{13,14}$. According to Task Force, inference is that therapeutic interventions that increase HRV may be protective against cardiac mortality and sudden cardiac death ${ }^{14}$.

\section{HR and RR interval (RRi) recording}

HR and RRi were recorded for posterior analysis of HRV at: 1) pre-operative day, for autonomic basal function characterization and 2) at discharge time. All recordings were obtained during the afternoon, at rest and, in a sitting position for $10 \mathrm{~min}$. Care was taken to avoid any manipulations during recording and no patient consumed caffeinated drinks/foods or smoked on the morning of assessments or during the procedure. Each patient rested for $10 \mathrm{~min}$ before the initiation of data collection to ensure the stabilization of HR.

\section{HRV analysis}

HR data were transferred to the computer and the RRi series were reviewed by visual inspection. Data were analyzed using Kubios HRV analysis software (MATLAB, version 2 beta, Kuopio, Finland). Series of 300 stable sequential RRi were considered for analysis.

Linear traditional measures in the time domain HRV analysis were evaluated by calculating the following, widely accepted parameters: Standard deviation of RRi (STD RR) in ms which represents a global index of HRV (overall HRV) and reflects all the cyclic components responsible for variability in the recording period; square root of the mean squared difference of successive RRi (RMSSD) in ms reflecting alterations in autonomic tone that are predominantly vagally mediated; and geometrical forms as the integral of the RRi histogram divided by the height of the histogram (RR tri index) and the baseline width of the RRi histogram (TINN) in ms, that provides an estimate of the overall HRV ${ }^{14-16}$.

\section{Statistical analysis}

Data are expressed as mean (standard deviation) unless otherwise specified. Between-group difference in continuous variables (pre-operative and discharge time) were examined by analysis of variance (ANOVA) or Kruskal-Wallis according to normality and/or equal variance, followed by Tukey's or Dunn's test. Fisher's Exact Test was applied for non-continuous variables. All the statistical analyses were two-tailed and $P$ values $\leq 0.05$ were considered significant. Statistical analyses were conducted using the SigmaPlot Software for Windows, version 11.0.

\section{Results}

Thirty patients were enrolled, and baseline characteristics are summarized in Table 1. No differences were observed among groups initially regarding clinical or demographic data, including age, height, gender and BMI. The surgical procedure data were comparable between groups, demonstrated by similar aortic cross clamping time, total surgery time and number of graft anastomoses. In addition, length of coronary care unit stay and post-operative hospital stay was also similar among groups. Usual medications prescribed after CABG were antiplatelet drug, statins, B-blockers, calcium antagonists, angiotensin converting enzyme inhibitors (ACEI) and analgesics. However, cardiopulmonary bypass time was different among the three groups with lower values in GEX2 compared to GEX1 and CG.

\section{Compliance with trial methodology}

All patients allocated in exercise groups, GEX1 and GEX2, participated in a once daily or twice-daily supervised postoperative exercise protocol, respectively, initiating on the first post-operative day until discharge (approximately five days). No clinically relevant adverse events occurred throughout the study. Vital signs remained within the range of normality in all patients.

\section{Heart rate variability}

There was no statistically significant difference among groups to HRV pre-operative condition (Table 2), although trends favoring GEX1 were observed (STD RR, RRtri, TINN, RMSSD). Postoperatively, at discharge, after rehabilitation completion program, HRV data demonstrated significant difference among the groups. Parasympathetic RMSSD HRV index and indexes of overall variability of RRi (STD RR, TINN) were significantly higher in GEX2 compared to the controls. In addition, mean HR was lower and mean RR was higher to exercise groups (GEX1 and GEX2), regardless dosage compared to $\mathrm{CG}$ as shown in Figure 1. However, there was no difference at discharge between the exercise groups (GEX1 and GEX2). 
Table 1. Basic clinical, risk factors and surgical data.

\begin{tabular}{|c|c|c|c|}
\hline & $\begin{array}{c}\text { CG } \\
\mathbf{n}=\mathbf{1 0}\end{array}$ & $\begin{array}{l}\text { GEX1 } \\
\mathbf{n}=10\end{array}$ & $\begin{array}{l}\text { GEX2 } \\
\mathrm{n}=10\end{array}$ \\
\hline Age, years & $56.8(10.8)$ & $59.3(7.9)$ & $58.1(4.9)$ \\
\hline Male, number [\%] & $9[90]$ & $8[80]$ & $8[80]$ \\
\hline Weight, kg & $72.8(11.1)$ & $71.9(14.7)$ & $70.6(9.6)$ \\
\hline Height, m & $1.63(0.06)$ & $1.66(0.07)$ & $1.65(0.09)$ \\
\hline Body mass index, $\mathrm{kg} / \mathrm{m}^{2}$ & $27.8(4.2)$ & $25.9(4.9)$ & $25.8(2.9)$ \\
\hline Respiratory rate, ipm & $18.1(4.2)$ & $18.2(1.5)$ & $19.7(3.0)$ \\
\hline \multicolumn{4}{|l|}{ Risk factors } \\
\hline Smoking history, number [\%] & $10[100]$ & $7[70]$ & $7[70]$ \\
\hline Arterial hypertension, number [\%] & $8[80]$ & $8[80]$ & $9[90]$ \\
\hline Diabetes mellitus, number [\%] & $5[50]$ & $6[60]$ & $6[60]$ \\
\hline \multicolumn{4}{|l|}{ Pharmacological treatment, number [\%] } \\
\hline$\beta$-blockers & $9[90]$ & $7[70]$ & $10[100]$ \\
\hline Calcium antagonists & - & - & $1[10]$ \\
\hline Antiplatelet & $10[100]$ & $10[100]$ & $10[100]$ \\
\hline \multicolumn{4}{|l|}{ Pre and post-operative data } \\
\hline Cardiopulmonary bypass, min & $77.5(11.3)$ & $72.0(25.8)$ & $49.2(17.7)^{\dagger \ddagger}$ \\
\hline Aortic cross clamping time, min & $41.2(8.0)$ & $40.6(18.9)$ & $29.1(13.2)$ \\
\hline Distal anastomoses, number & $2.5(0.8)$ & $2.6(0.8)$ & $2.1(0.6)$ \\
\hline Coronary care unit stay, days & $2.2(0.8)$ & $2.0(0.0)$ & $2.5(1.3)$ \\
\hline Post-operative hospital stay, days & $4.9(1.7)$ & $4.9(0.3)$ & $5.0(1.1)$ \\
\hline
\end{tabular}

Data presented as mean (standard deviation, SD) or number (percentage) of subjects; $\mathrm{CG}=$ Control Group; GEX1 = one exercise daily session; GEX2 = two exercise daily sessions; † statistical difference between CG and GEX2; †statistical difference between GEX1 and GEX2.

Table 2. Heart rate variability indexes at baseline and discharge.

\begin{tabular}{|c|c|c|c|c|}
\hline & $\begin{array}{c}\text { CG } \\
n=10\end{array}$ & $\begin{array}{l}\text { GEX1 } \\
n=10\end{array}$ & $\begin{array}{l}\text { GEX2 } \\
\mathrm{n}=10\end{array}$ & $P$-value \\
\hline \multicolumn{5}{|c|}{ Mean RR, ms } \\
\hline Baseline & 810.3 (153.9) & $939.21(86.4)$ & 904.8 (110.9) & 0.14 \\
\hline Discharge & $602.8(59.1)$ & $759.1(57.0)^{*}$ & $694.0(101.8)^{*}$ & 0.0004 \\
\hline \multicolumn{5}{|c|}{ Mean HR, bpm } \\
\hline Baseline & $76.7(15.0)$ & $64.4(6.0)$ & $67.1(7.4)$ & 0.14 \\
\hline Discharge & $100.4(10.1)$ & $79.4(5.7)^{*}$ & $88.1(12.3)^{*}$ & 0.0002 \\
\hline \multicolumn{5}{|c|}{ STD RR, ms } \\
\hline Baseline & $17.5(7.2)$ & $20.1(6.3)$ & $13.5(2.6)$ & 0.07 \\
\hline Discharge & $4.8(2.1)$ & $7.0(1.6)$ & $7.6(3.9)^{*}$ & 0.05 \\
\hline \multicolumn{5}{|c|}{ RMSSD, ms } \\
\hline Baseline & $12.0(4.4)$ & $20.4(9.2)$ & $13.1(5.6)$ & 0.06 \\
\hline Discharge & $4.6(1.8)$ & $6.9(1.2)$ & $7.9(4.6)^{*}$ & 0.04 \\
\hline \multicolumn{5}{|l|}{ RR Tri } \\
\hline Baseline & $5.1(1.9)$ & $6.1(1.9)$ & $4.2(0.7)$ & 0.07 \\
\hline Discharge & $1.9(0.3)$ & $2.3(0.4)$ & $2.6(0.4)$ & 0.13 \\
\hline \multicolumn{5}{|l|}{ TINN, ms } \\
\hline Baseline & $84.0(35.6)$ & $96.5(30.2)$ & $65.5(15.5)$ & 0.06 \\
\hline Discharge & $23(10.6)$ & $35(7.4)$ & $38(21.2)^{*}$ & 0.04 \\
\hline
\end{tabular}

Data presented as mean (standard deviation, $\mathrm{SD}$ ); $\mathrm{CG}=$ Control Group; GEX1 = one exercise daily session; GEX2 = two exercise daily sessions; RR = R-R intervals; $\mathrm{STD}$ $\mathrm{RR}=$ standard deviation of all RR; HR = heart rate; RMSSD = square root of the mean squared difference of successive RR; RRTRI = triangular index; $\mathrm{TINN}=$ triangular interpolation of RR intervals.

\section{Discussion}

This study aimed to investigate a potential dose-response of an exercise-based impatient program after cardiac CABG surgery on CAM. Our results suggest that regardless the doses of exercise (once or twice a day) there was significant cardiac autonomic benefit compared to controls.
Dose-response of physical activity and health benefits has been demonstrated for overall mortality, risk of coronary heart disease and numerous health outcomes including cardiorespiratory fitness, body composition and high-density lipoprotein cholesterol (HDL-C) in diverse populations ${ }^{17-21}$. In postmenopausal women, Friedenreich et al. ${ }^{19}$ observed 

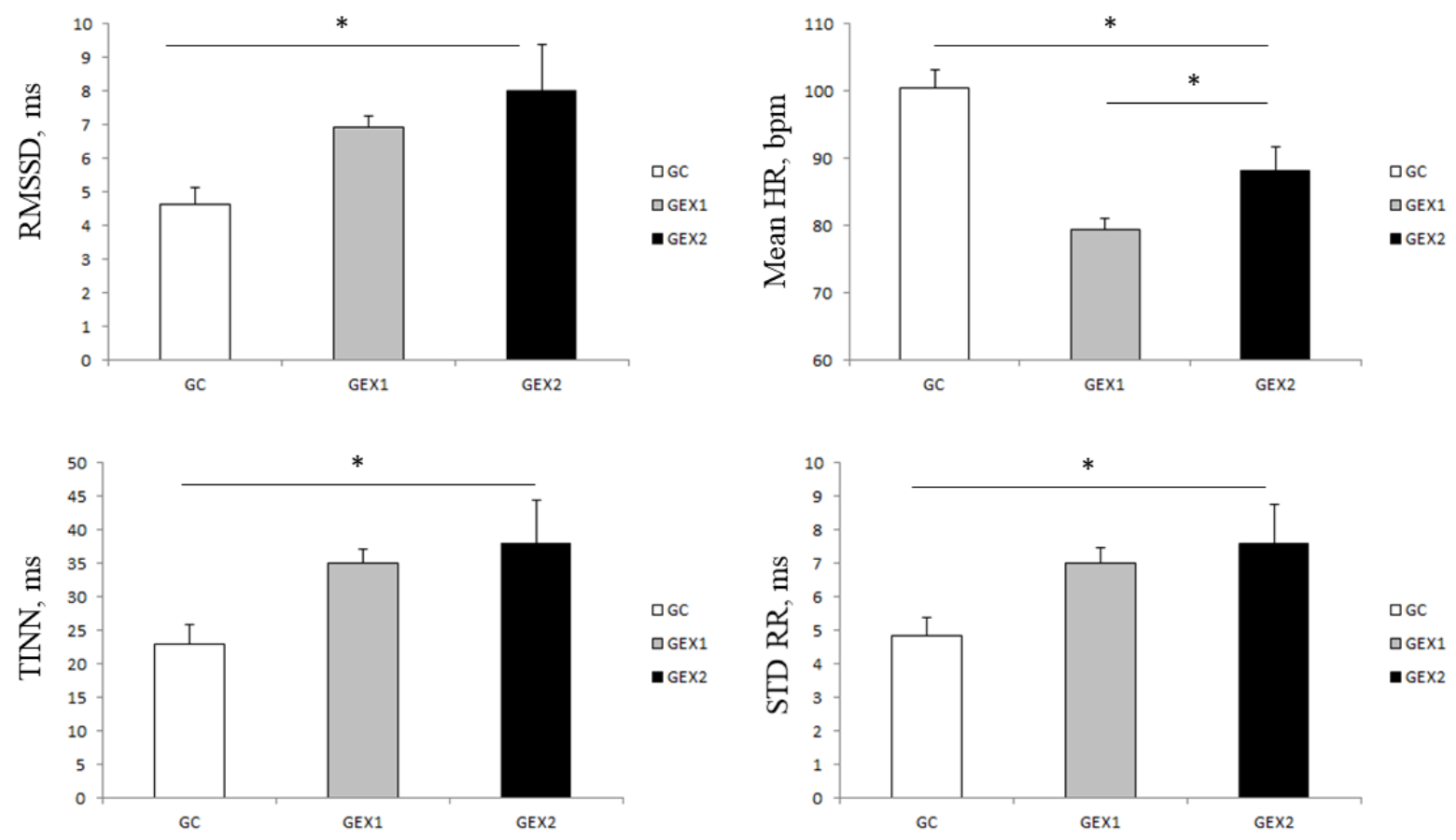

Figure 1. HRV indexes at discharge. Abbreviations: STD RR: standard deviation of the mean of all inter-beat intervals; RRtri: triangular index; TI NN: triangular interpolation of NN interval histogram; RMSSD: square root of the mean squared difference of successive RR intervals, ms: milliseconds; $H R=$ heart rate; $C G=$ Control Group; GEX1 = one exercise daily session; GEX2 = two exercise daily sessions. ${ }^{*}$ Different from $\mathrm{CG}$.

an additional benefit of higher-volume aerobic exercise (300 versus 150 minutes/week) for adiposity outcomes and risk of postmenopausal breast cancer, while Lee et al. ${ }^{21}$ showed that elderly men who exercised once or twice per week had a lower risk of all-cause mortality compared with sedentary.

Although these studies have supported the dose-response relationship with largest health improvements among training programs of greater duration and/or intensity ${ }^{22,23}$, there is no study involving patients after cardiac surgery, more specifically in CABG surgery, and exercise programs performed during the hospitalization phase (CR phase I).

Early mobilization and exercise-based inpatient rehabilitation after cardiac surgery has gained wide acceptance and evidence ${ }^{2}$. A multifactorial positive effect value has been proved, including effects on postoperative respiratory complications, functional capacity and $\mathrm{CAM}^{2,5,6,9,24-26}$. Previously, our group was able to demonstrate a positive cardiac autonomic effect of an early mobilization program performed once a day ${ }^{5,6}$. At hospital discharge, patients that engaged in an early mobilization protocol presented higher values of parasympathetic and overall $\mathrm{HRV}$ indexes compared to a control group 5 .

Although some effect of early mobilization on HRV has already been demonstrated, no information about the dosage of exercise intervention is available at this early stage of rehabilitation. The current study is the first to focus directly on the influence of different dosages of an inpatient exercise program on HRV parameters in patients who underwent cardiac surgery.

For this purpose, in a comparison of once or twice a day and no systematic rehabilitation, the group enrolled to physical exercise twice-daily demonstrated significantly higher values of overall variability indexes of RRi (STD RR and TINN) and parasympathetic status (as shown by greater HRV RMSSD index) compared to control group. On the other hand, mean HR presented lower values at discharge regardless of the volume of exercise imposed with significant difference for both groups (once and twice a day) in comparison to control group. Therefore, our current results suggest that both doses of exercise provided cardiac autonomic benefits without advantage.

To highlight the clinical importance of these findings, it is relevant to point out the role of the autonomic nervous system in the regulation of the cardiovascular system in order to match cardiac output with the body's need during various exogenic stimuli ${ }^{14}$. Moreover, cardiac autonomic nervous system has a significant impact on cardiac electrophysiology and arrhythmogenesis, which is an important clinical aspect mainly after a surgical procedure $e^{27,28}$.

The finding of high resting heart rate of this study was proved to be associated with ventricular arrhythmogenesis 
(positive ventricular late potentials) by factors as premature ventricular complexes and depressed HRV ${ }^{29}$. It was also previously demonstrated that a decreased HRV may be indicative of an electrically unstable myocardium ${ }^{28}$, myocardial vulnerability and occurrence of malignant arrhythmias ${ }^{14}$. Besides that, it has been demonstrated that CABG patients with postoperative decreased HRV have a higher incidence of adverse coronary events, such as new myocardial infarction or sudden death ${ }^{8,30}$. In this study, longitudinal follow-up was not performed to investigate these outcomes, which would be interesting in future studies.

Inpatient and outpatient exercise-based programs have been shown to increase HRV and consequently the cardiac autonomic regulation in a variety of clinical populations ${ }^{31-33}$. A similar hospital-based exercise program was carried out post-acute myocardium infarction once a day and resulted in increased vagal and decreased sympathetic cardiac modulation; however, exercise dosage was not the focus of that study ${ }^{34}$.

In the outpatient field, some evidence suggests that at least two mediators, angiotensin II and nitric oxide, may play roles in mechanisms contributing to beneficial modifications of $\mathrm{HRV}^{33}$. Nitric oxide has a direct effect on vagal tone $\mathrm{e}^{35}$ and angiotensin is an inhibitor of cardiac vagal activity $^{36}$. However, there is no studies available in which the mechanisms responsible to improve cardiac regulation after CABG facing early mobilization were investigated.

Previous studies showed that surgical procedure per se leads to an impairment of cardiac autonomic regulation attributed to several factors such as direct surgical manipulation of the heart and adjacent structures, duration of surgery, anesthesia, aortic clamping, cardioplegic arrest and cardiopulmonary bypass extracorporeal circulation $(\mathrm{CPB})^{37,38}$. In the current study, although CPB time was shorter in GEX2, authors conducted an additional association analysis between HRV indexes versus $\mathrm{CPB}$ time and there was no relationship between them, suggesting there are no effects of this surgical factor on the studied sample.

A strength of this study was to show an import and practical aspect regarding one and two exercises daily sessions and no influence of inpatient exercise dosage on cardiac autonomic adaptation after CABG. Importantly, the terminus dose can be referred to different components of exercise training such as frequency, intensity, and duration. In the present study, distinct dosage was considered by adjusting frequency of exercise, adding a complete exercise session in another period of the day. Thus, there are several possibilities to change and test dose-response in exercise rehabilitation programs which deserves attention in results' comparisons.

Despite its strength, the study has limitations. The small sample, which may have contributed to the absence of difference between groups due to a Type II error. The study design did not consider an intention to treat analysis and only those who actually performed all the proposed steps were considered to be part of the final analysis. The quasiexperimental design with non-random allocation is liable to bias; however, it was the only way to develop the study due to logistical factors. In addition, HRV assessments did not consider the effects of respiratory rate and daily medications. Furthermore, due to the harmful potential of surgical procedure to autonomic system, baseline parameters should ideally be considered in the period after the surgery. Finally, we have to assume a potential influence of cardiopulmonary bypass time on cardiac autonomic modulation, although sub-analysis in the present study have not pointed that out.

In conclusion, our results suggest that regardless dosage, post-CABG patients who engaged in a mobilization-exercise program demonstrated significant better cardiac autonomic modulation at discharge compared with the control group. The results are encouraging further investigation in a more comprehensive sample to reinforce the findings in the field of in-hospital exercise rehabilitation investigation.

\section{Funding}

This study was supported by a Research Grant from Fundação de Amparo à Pesquisa do Estado de São Paulo (FAPESP), grant numbers 05/59427-7; 09/54194-5; 15/12763-4.

\section{Conflict of interest}

None

\section{Acknowledgements}

We thank all colleagues from the Cardiopulmonary Physiotherapy Laboratory and the staff at the Department of Cardiac Surgery of Irmandade Santa Casa Misericordia Hospital of Araraquara for their support during the trial. We thank all the patients who participated in the study for their valuable input.

\section{References}

1. Neumann FJ, Sousa-Uva M, Ahlsson A, Alfonso F, Banning AP, Benedetto U, et al. 2018 ESC/EACTS Guidelines on myocardial Revascularization. Eur Heart J. 2019;40(2):87-165. http://dx.doi. org/10.1093/eurheartj/ehy394. PMid:30165437.

2. Santos PMR, Ricci NA, Suster ÉAB, Paisani DM, Chiavegato LD. Effects of early mobilisation in patients after cardiac surgery: a systematic review. Physiotherapy. 2017 Mar;103(1):1-12. http://dx.doi. org/10.1016/j.physio.2016.08.003. PMid:27931870.

3. Herdy AH, Marcchi PL, Vila A, Tavares C, Collaço J, Niebauer J, et al. Pre- and postoperative cardiopulmonary rehabilitation in hospitalized patients undergoing coronary artery bypass surgery: a randomized controlled trial. Am J Phys Med Rehabil. 2008;87(9):714-9. http:// dx.doi.org/10.1097/PHM.0b013e3181839152. PMid:18716482.

4. Monteleone S, Dalla Toffola E, Emiliani V, Ricotti S, Bruggi M, Conte $\mathrm{T}$, et al. Recovery of deambulation after cardiothoracic surgery: a single center experience. Eur J Phys Rehabil Med. 2015;51(6):763-71. PMid:25800502.

5. Mendes RG, Simões RP, Costa FDSM, Pantoni CBF, Di Thommazo L, Luzzi S, et al. Short-term supervised inpatient physiotherapy exercise protocol improves cardiac autonomic function after coronary artery bypass graft surgery - a randomised controlled trial. Disabil Rehabil. 
2010;32(16):1320-7. http://dx.doi.org/10.3109/09638280903483893. PMid:20156053.

6. Pantoni CB, Mendes RG, Di Thommazo-Luporini L, Simões RP, Amaral-Neto O, Arena R, et al. Recovery of linear and nonlinear heart rate dynamics after coronary artery bypass grafting surgery. Clin Physiol Funct Imaging. 2014;34(6):449-56. http://dx.doi.org/10.1111/ cpf.12115. PMid:24666784.

7. Laitio TT, Mäkikallio TH, Huikuri HV, Kentala ES, Uotila P, Jalonen JR, et al. Relation of heart rate dynamics to the occurrence of myocardial ischemia after coronary artery bypass grafting. Am J Cardiol. 2002;89(10):1176-81. http://dx.doi.org/10.1016/S00029149(02)02300-7. PMid:12008171.

8. Lakusic N, Mahovic D, Kruzliak P, Cerkez Habek J, Novak M, Cerovec D. Changes in heart rate variability after coronary artery bypass grafting and clinical importance of these findings. BioMed Res Int. 2015;2015:680515. http://dx.doi.org/10.1155/2015/680515. PMid:26078960.

9. Westerdahl E, Möller M. Physiotherapy-supervised mobilization and exercise following cardiac surgery: a national questionnaire survey in Sweden. J Cardiothorac Surg. 2010;5(1):67. http://dx.doi. org/10.1186/1749-8090-5-67. PMid:20738852.

10. Vidoni ED, Johnson DK, Morris JK, Van Sciver A, Greer CS, Billinger SA, et al. Dose-Response of Aerobic Exercise on Cognition: A Community-Based, Pilot Randomized Controlled Trial. PLoS One. 2015;10(7):e0131647. http://dx.doi.org/10.1371/journal. pone.0131647. PMid:26158265.

11. Zech A, Hendrich S, Pfeifer K. Association between exercise therapy dose and functional improvements in the early postoperative phase after hip and knee arthroplasty: an observational study. PM R. 2015;7(10):1064-72. http://dx.doi.org/10.1016/j.pmrj.2015.04.008. PMid:25892356.

12. Papathanasiou G, Tsamis N, Georgiadou P, Adamopoulos S. Beneficial effects of physical training and methodology of exercise prescription in patients with heart failure. Hellenic J Cardiol. 2008;49(4):267-77. PMid:18935714.

13. Catai A, Pastre C, Godoy M, Silva E, Takahashi A, Vanderlei L. Heart rate variability: are you using it properly? Standardisation checklist of procedures. Braz J Phys Ther. 2020;24(2):91-102. http://dx.doi. org/10.1016/j.bjpt.2019.02.006. PMid:30852243.

14. Variability HR. Standards of measurement, physiological interpretation and clinical use. Task Force of the European Society of Cardiology and the North American Society of Pacing and Electrophysiology. Circulation. 1996;93(5):1043-65. PMid:8598068.

15. Vanderlei LCM, Pastre CM, Hoshi RA, Carvalho TD, Godoy MF. Basic notions of heart rate variability and its clinical applicability. Rev Bras Cir Cardiovasc. 2009;24(2):205-17. http://dx.doi.org/10.1590/ S0102-76382009000200018. PMid:19768301.

16. Shaffer F, Ginsberg JP. An overview of heart rate variability metrics and norms. Front Public Health. 2017 Sep 28;5:258. http://dx.doi. org/10.3389/fpubh.2017.00258. PMid:29034226.

17. Dalleck LC, Allen BA, Hanson BA, Borresen EC, Erickson ME, De Lap SL. Dose-response relationship between moderate-intensity exercise duration and coronary heart disease risk factors in postmenopausal women. J Womens Health. 2009;18(1):105-13. http://dx.doi. org/10.1089/jwh.2008.0790. PMid:19132882.

18. Foulds HJ, Bredin SS, Charlesworth SA, Ivey AC, Warburton DE. Exercise volume and intensity: a dose-response relationship with health benefits. Eur J Appl Physiol. 2014;114(8):1563-71. http://dx.doi. org/10.1007/s00421-014-2887-9. PMid:24770699.

19. Friedenreich CM, Neilson HK, O'Reilly R, Duha A, Yasui Y, Morielli $\mathrm{AR}$, et al. Effects of a high vs moderate volume of aerobic exercise on adiposity outcomes in postmenopausal women: a randomized clinical trial. JAMA Oncol. 2015;1(6):766-76. http://dx.doi.org/10.1001/ jamaoncol.2015.2239. PMid:26181634.

20. Lee IM, Sesso HD, Oguma Y, Paffenbarger RS Jr. Relative Intensity of Physical Activity and Risk of Coronary Heart Disease. Circulation. 2003;107(8):1110-6. http://dx.doi.org/10.1161/01. CIR.0000052626.63602.58. PMid:12615787.

21. Lee IM, Skerrett PJ. Physical activity and all-cause mortality: what is the dose-response relation? Med Sci Sports Exerc. 2001;33(6, Suppl.):S459-94. http://dx.doi.org/10.1097/00005768-20010600100016. PMid:11427772.

22. Warburton DE, Charlesworth S, Ivey A, Nettlefold L, Bredin SS. A systematic review of the evidence for Canada's Physical Activity Guidelines for Adults. Int J Behav Nutr Phys Act. 2010;7(1):39. http:// dx.doi.org/10.1186/1479-5868-7-39. PMid:20459783.

23. Woodlief TL, Carnero EA, Standley RA, Distefano G, Anthony SJ, Dubis GS, et al. Dose response of exercise training following rouxen-Y gastric bypass surgery: a randomized trial. Obesity (Silver Spring). 2015;23(12):2454-61. http://dx.doi.org/10.1002/oby.21332. PMid:26537198.

24. Anderson L, Oldridge N, Thompson DR, Zwisler AD, Rees K, Martin N, et al. Exercise-based cardiac rehabilitation for coronary heart disease: cochrane systematic review and meta-analysis. J Am Coll Cardiol. 2016;67(1):1-12. http://dx.doi.org/10.1016/j. jacc.2015.10.044. PMid:26764059.

25. Mendes RG, Simões RP, Costa FSM, Pantoni CB, Di Thommazo L, Luzzi S, et al. Left-ventricular function and autonomic cardiac adaptations after short-term inpatient cardiac rehabilitation: a prospective clinical trial. J Rehabil Med. 2011;43(8):720-7. http:// dx.doi.org/10.2340/16501977-0843. PMid:21732006.

26. Pantoni CB, Di Thommazo-Luporini L, Mendes RG, Caruso FC, Mezzalira D, Arena R, et al. continuous positive airway pressure during exercise improves walking time in patients undergoing inpatient cardiac rehabilitation after coronary artery bypass graft surgery: a randomized controlled trial. J Cardiopulm Rehabil Prev. 2016;36(1):20-7. http:// dx.doi.org/10.1097/HCR.0000000000000144. PMid:26468628.

27. Huikuri HV. Heart rate dynamics and vulnerability to ventricular tachyarrhythmias. Ann Med. 1997;29(4):321-5. http://dx.doi. org/10.3109/07853899708999355. PMid:9375990.

28. Shen MJ, Zipes DP. Role of the autonomic nervous system in modulating cardiac arrhythmias. Circ Res. 2014;114(6):1004-21. http:// dx.doi.org/10.1161/CIRCRESAHA.113.302549. PMid:24625726.

29. Soliman EZ, Elsalam MA, Li T. The relationship between high resting heart rate and ventricular arrhythmogenesis in patients referred to ambulatory $24 \mathrm{~h}$ electrocardiographic recording. EP Europace. 2010;12(2):261-5. http://dx.doi.org/10.1093/europace/eup344. PMid:19887457.

30. Ksela J, Suwalski P, Kalisnik JM, Avbelj V, Suwalski G, Gersak B. Assessment of Nonlinear Heart Rate Dynamics after Beating-Heart Revascularization. Heart Surg Forum. 2009;12(1):E10-6. http://dx.doi. org/10.1532/HSF98.20081116. PMid:19233759.

31. Caruso FR, Arena R, Phillips SA, Bonjorno JC Jr, Mendes RG, Arakelian VM, et al. Resistance exercise training improves heart rate variability and muscle performance: a randomized controlled trial in coronary artery disease patients. Eur J Phys Rehabil Med. 2015;51(3):281-9. PMid:25384514.

32. Murad K, Brubaker PH, Fitzgerald DM, Morgan TM, Goff DC Jr, Soliman EZ, et al. Exercise training improves heart rate variability in older patients with heart failure: a randomized, controlled, singleblinded trial. Congest Heart Fail. 2012;18(4):192-7. http://dx.doi. org/10.1111/j.1751-7133.2011.00282.x. PMid:22536936.

33. Routledge FS, Campbell TS, McFetridge-Durdle JA, Bacon SL. Improvements in heart rate variability with exercise therapy. Can 
J Cardiol. 2010;26(6):303-12. http://dx.doi.org/10.1016/S0828282X(10)70395-0. PMid:20548976.

34. Santos-Hiss MD, Melo RC, Neves VR, Hiss FC, Verzola RM, Silva E, et al. Effects of progressive exercise during phase I cardiac rehabilitation on the heart rate variability of patients with acute myocardial infarction. Disabil Rehabil 2011;33(10):835-42. http://dx.doi.org/10.3109/09638288.2010 514016. PMid:20809873.

35. Chowdhary S, Townend JN. Role of nitric oxide in the regulation of cardiovascular autonomic control. Clin Sci. 1999;97(1):5-17. http:// dx.doi.org/10.1042/CS19980399. PMid:10369789.
36. Townend JN, al-Ani M, West JN, Littler WA, Coote JH. Modulation of cardiac autonomic control in humans by angiotensin II. Hypertension. 1995;25(6):1270-5. http://dx.doi.org/10.1161/01.HYP.25.6.1270. PMid:7768573.

37. Komatsu T, Kimura T, Sanchala V, Shibutani K, Shimada Y. Effects of fentanyl-diazepam-pancuronium anesthesia on heart rate variability: a spectral analysis. J Cardiothorac Vasc Anesth. 1992;6(4):444-8. http:// dx.doi.org/10.1016/1053-0770(92)90011-U. PMid:1498300.

38. Murphy DA, Armour JA. Influences of cardiopulmonary bypass, temperature, cardioplegia, and topical hypothermia on cardiac innervation. J Thorac Cardiovasc Surg. 1992;103(6):1192-9. http:// dx.doi.org/10.1016/S0022-5223(19)34887-1. PMid:1597985. 


\section{Author contributions}

Conception of the work: RGM, CBFP, ABS; Acquisition: RGM, CBFP, RPS, LDL, FCRCB; Analysis and interpretation of data: All authors; Draft and revision of the work: All authors; Final approval of the version to be published: All authors; Awareness of all aspects of work: All authors.

\section{Author information}

\section{Renata Gonçalves Mendes}

Graduated in Physiotherapy, Araraquara University Center (UNIARA), SP, Brazil; PhD in

Physiotherapy, Federal University of São Carlos (UFSCar), SP, Brazil; Adjunct Professor, Department of Physiotherapy, Federal University of São Carlos (UFSCar), SP, Brazil.

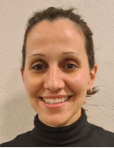

\section{Camila Bianca Falasco Pantoni}

Graduated in Physiotherapy, Department of Physiotherapy Federal University of São Carlos (UFSCar), SP, Brazil; PhD in Physiotherapy, Federal University of São Carlos (UFSCar), SP, Brazil; Adjunct Professor, Gerontology Department, Federal University of São Carlos (UFSCar), SP, Brazil.

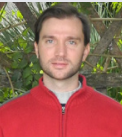

\section{Rodrigo Polaquini Simões}

Graduated in Physiotherapy, Camilo Castelo Branco University (Unicastelo), SP, Brazil; PhD in Physiotherapy, Federal University of São Carlos (UFSCar), SP, Brazil; Professor in Rehabilitation Sciences, Federal University of Alfenas, UNIFAL, MG, Brazil.

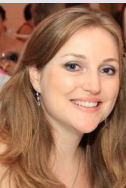

Luciana Ditomaso-Luporini

Graduated in Physiotherapy, Department of Physiotherapy Federal University of São Carlos (UFSCar), SP, Brazil; PhD in Physiotherapy, Federal University of São Carlos (UFSCar), SP, Brazil; Multiprofessional Team Manager, Irmandade Santa Casa Misericordia Hospital of Sao Carlos, SP, Brazil

Flávia Cristina Rossi Caruso Bonjorno

Graduated in Physiotherapy, Araraquara University Center (UNIARA), SP, Brazil; Specialization in Exercise Physiology, Federal University of São Carlos (UFSCar); PhD in Physiotherapy, Federal University of São Carlos (UFSCar), SP, Brazil

\section{Erika Zavaglia Kabbach}

Graduated in Physiotherapy, Department of Physiotherapy Federal University of São Carlos (UFSCar), SP, Brazil; PhD in Physiotherapy, Federal University of São Carlos (UFSCar), SP, Brazil; Physiotherapist at Americo Brasiliense Stadual Hospital (HEAB), SP, Brazil.

\section{Audrey Borghi-Silva}

Graduated in Physiotherapy, Londrina State University (UEL) PR, Brazil; PhD in Physiotherapy, Federal University of São Carlos (UFSCar), SP, Brazil; Full Professor, Department of Physiotherapy Federal University of São Carlos (UFSCar), SP, Brazil. 